\title{
Lower Bounds for Numbers of Real Self-Dual Spaces in Problems of Schubert Calculus
}

\author{
Kang $L U$
}

Department of Mathematical Sciences, Indiana University - Purdue University Indianapolis, 402 North Blackford St, Indianapolis, IN 46202-3216, USA

E-mail: lukang@iupui.edu

Received November 27, 2017, in final form May 07, 2018; Published online May 14, 2018

https://doi.org/10.3842/SIGMA.2018.046

\begin{abstract}
The self-dual spaces of polynomials are related to Bethe vectors in the Gaudin model associated to the Lie algebras of types B and C. In this paper, we give lower bounds for the numbers of real self-dual spaces in intersections of Schubert varieties related to osculating flags in the Grassmannian. The higher Gaudin Hamiltonians are self-adjoint with respect to a nondegenerate indefinite Hermitian form. Our bound comes from the computation of the signature of this form.
\end{abstract}

Key words: real Schubert calculus; self-dual spaces; Bethe ansatz; Gaudin model

2010 Mathematics Subject Classification: 14N99; 17B80; 82B23

\section{Introduction}

It is well known that the problem of finding the number of real solutions to algebraic systems is very difficult, and not many results are known. In particular, the counting of real points in problems of Schubert calculus in the Grassmannian has received a lot of attention, see [2, 5, 6, $7,13,19,20]$ for example. In this paper, we give lower bounds for the numbers of real self-dual spaces in intersections of Schubert varieties related to osculating flags in the Grassmannian.

We define the Grassmannian $\operatorname{Gr}(N, d)$ to be the set of all $N$-dimensional subspaces of the $d$-dimensional space $\mathbb{C}_{d}[x]$ of polynomials in $x$ of degree less than $d$. In other words, we always assume for $X \in \operatorname{Gr}(N, d)$, we have $X \subset \mathbb{C}_{d}[x]$. Set $\mathbb{P}^{1}=\mathbb{C} \cup\{\infty\}$. Then, for any $z \in \mathbb{P}^{1}$, we have the osculating flag $\mathcal{F}(z)$, see (4.1), (4.2). Denote the Schubert cells corresponding to $\mathcal{F}(z)$ by $\Omega_{\xi}(\mathcal{F}(z))$, where $\xi=\left(d-N \geqslant \xi_{1} \geqslant \xi_{2} \geqslant \cdots \geqslant \xi_{N} \geqslant 0\right)$ are partitions. Then the set $\Omega_{\boldsymbol{\xi}, \boldsymbol{z}}$ consists of spaces $X \in \operatorname{Gr}(N, d)$ such that $X$ belongs to the intersection of Schubert cells $\Omega_{\xi^{(i)}}\left(\mathcal{F}\left(z_{i}\right)\right)$ for $\boldsymbol{z}=\left(z_{1}, \ldots, z_{n}\right)$ and $\boldsymbol{\xi}=\left(\xi^{(1)}, \ldots, \xi^{(n)}\right)$, where all $z_{i} \in \mathbb{P}^{1}$ are distinct and $\xi^{(i)}$ are partitions, see (4.3). A point $X \in \operatorname{Gr}(N, d)$ is called real if it has a basis consisting of polynomials with all coefficients real. A lower bound for the number of real points in $\Omega_{\boldsymbol{\xi}, \boldsymbol{z}}$ is given in [13].

Let $X \in \operatorname{Gr}(N, d)$ be an $N$-dimensional subspace of polynomials in $x$. Let $X^{\vee}$ be the $N$ dimensional space of polynomials which are Wronskian determinants of $N-1$ elements of $X$

$$
X^{\vee}=\left\{\operatorname{det}\left(d^{i-1} \varphi_{j} / d x^{i-1}\right)_{i, j=1}^{N-1}, \varphi_{j}(x) \in X\right\}
$$

The space $X$ is called self-dual if $X^{\vee}=\psi \cdot X$ for some polynomial $\psi(x)$, see [16]. We define $\mathrm{s} \Omega_{\boldsymbol{\xi}, \boldsymbol{z}}$ the subset of $\Omega_{\xi, z}$ consisting of all self-dual spaces. Our main result of this paper is a lower bound for the number of real self-dual spaces in $\Omega_{\boldsymbol{\xi}, \boldsymbol{z}}$, see Corollary 7.4, i.e., a lower bound for the number of real points in $\mathrm{s} \Omega_{\boldsymbol{\xi}, \boldsymbol{z}}$, by following the idea of [13].

Let $\mathfrak{g}_{N}$ be the Lie algebra $\mathfrak{s o}_{2 r+1}$ if $N=2 r$ or the Lie algebra $\mathfrak{s p}_{2 r}$ if $N=2 r+1$. We also set $\mathfrak{g}_{3}=\mathfrak{s l}_{2}$. It is known from [10], see also [16, Section 6.1], that if $\mathrm{s} \Omega_{\boldsymbol{\xi}, \boldsymbol{z}}$ is nonempty, then 
$\xi_{i}^{(s)}-\xi_{i+1}^{(s)}=\xi_{N-i}^{(s)}-\xi_{N-i+1}^{(s)}$ for $i=1, \ldots, N-1$. Hence the $\mathfrak{s l}_{N}$-weight corresponding to the partition $\xi^{(s)}$ has certain symmetry and thus induces a $\mathfrak{g}_{N}$-weight $\lambda^{(s)}$, cf. (4.4). Therefore, the sequence of partitions $\boldsymbol{\xi}$ with nonempty s $\Omega_{\boldsymbol{\xi}, \boldsymbol{z}}$ can be expressed in terms of a sequence of dominant integral $\mathfrak{g}_{N^{-}}$weights $\boldsymbol{\lambda}=\left(\lambda^{1}, \ldots, \lambda^{(n)}\right)$ and a sequence of nonnegative integers $\boldsymbol{k}=\left(k_{1}, \ldots, k_{n}\right)$, see Lemma 4.1. In particular, $k_{i}=\xi_{N}^{(i)}$. We call $\boldsymbol{\xi}, \boldsymbol{z}$ or $\boldsymbol{\lambda}, \boldsymbol{k}, \boldsymbol{z}$ the ramification data.

As a subset of $\Omega_{\boldsymbol{\xi}, \boldsymbol{z}}, \mathrm{s} \Omega_{\boldsymbol{\xi}, \boldsymbol{z}}$ can be empty even if $\Omega_{\boldsymbol{\xi}, \boldsymbol{z}}$ is infinite. However, if $\mathrm{s} \Omega_{\boldsymbol{\xi}, \boldsymbol{z}}$ is nonempty, then $\mathrm{s} \Omega_{\boldsymbol{\xi}, \boldsymbol{z}}$ is finite if and only if $\Omega_{\boldsymbol{\xi}, \boldsymbol{z}}$ is finite. More precisely, if

$$
|\boldsymbol{\xi}|:=\sum_{i=1}^{n}\left|\xi^{(i)}\right|=N(d-N),
$$

then the number of points in $\mathrm{s} \Omega_{\boldsymbol{\xi}, \boldsymbol{z}}$ counted with multiplicities equals the multiplicity of the trivial $\mathfrak{g}_{N^{-}}$-module in the tensor product $V_{\lambda^{(1)}} \otimes \cdots \otimes V_{\lambda^{(n)}}$ of irreducible $\mathfrak{g}_{N^{-}}$-modules of highest weights $\lambda^{(1)}, \ldots, \lambda^{(n)}$. Since we are interested in the counting problem, from now on, we always assume that $|\boldsymbol{\xi}|=N(d-N)$.

For brevity, we consider $\infty$ to be real. If all $z_{1}, \ldots, z_{n}$ are real, it follows from [14, Theorem 1.1] that all points in $\mathrm{s} \Omega_{\boldsymbol{\xi}, \boldsymbol{z}}$ are real. Hence the number of real points is maximal possible in this case. Moreover, it follows from [15, Corollary 6.3] that all points in $\mathrm{s} \Omega_{\boldsymbol{\xi}, \boldsymbol{z}}$ are multiplicity-free.

Then we want to know how many real points we can guarantee in other cases. In general, a necessary condition for the existence of real points is that the set $\left\{z_{1}, \ldots, z_{n}\right\}$ should be invariant under the complex conjugation and the partitions at the complex conjugate points are the same. In other words, $\left(\lambda^{(i)}, k_{i}\right)=\left(\lambda^{(j)}, k_{j}\right)$ provided $z_{i}=\bar{z}_{j}$. In this case we say that $\boldsymbol{z}, \boldsymbol{\lambda}, \boldsymbol{k}$ are invariant under conjugation. Moreover, the greatest common divisor of $X \in \mathrm{s} \Omega_{\xi, z}$ in this case is a real polynomial. Hence we reduce the problem to the case that $k_{i}=0$, for all $i=1, \ldots, n$.

The derivation of the lower bounds is based on the identification of the self-dual spaces of polynomials with points of spectrum of higher Gaudin Hamiltonians of types B and C ( $\mathfrak{g}_{N}$, $N \geqslant 4$ ) built in [10] and [16], see Theorem 5.2. We show that higher Gaudin Hamiltonians of types B and C have certain symmetry with respect to the Shapovalov form which is positive definite Hermitian, see Proposition 6.1. In particular, these operators are self-adjoint with respect to the Shapovalov form for real $z_{1}, \ldots, z_{n}$ and hence have real eigenvalues. Therefore, it follows from Theorem 5.2 that self-dual spaces with real $z_{1}, \ldots, z_{n}$ are real.

If some of $z_{1}, \ldots, z_{n}$ are not real, but the data $\boldsymbol{z}, \boldsymbol{\lambda}, \boldsymbol{k}$ are invariant under the complex conjugation, the higher Gaudin Hamiltonians are self-adjoint with respect to a nondegenerate (indefinite) Hermitian form. One of the key observations for computing the lower bound for the number of real points in $s \Omega_{\boldsymbol{\xi}, \boldsymbol{z}}$ is the fact that the number of real eigenvalues of such operators is at least the absolute value of the signature of the Hermitian form, see Lemma 6.4.

The computation of the signature of the form is reduced to the computation of the character values of products of symmetric groups on products of commuting transpositions. The formula for such character, similar to the Frobenius formula in [4] and [13, Proposition 2.1], is given in Proposition 3.1. Consequently, we obtain our main result, a lower bound for the number of real points in $\mathrm{s} \Omega_{\boldsymbol{\xi}, \boldsymbol{z}}$ for $N \geqslant 4$, see Corollary 7.4. The case $N=2$ is the same as that of [13] since every 2-dimensional space of polynomials is self-dual. By the proof of [10, Theorem 4.19], the case $N=3$ is reduced to the case of [13], see Section 7.2.

Based on the identification of the self-self-dual spaces of polynomials with points of spectrum of higher Gaudin Hamiltonians of type $\mathrm{G}_{2}$ built in [1] and [8], we expect that lower bounds for the numbers of real self-self-dual spaces in $\Omega_{\boldsymbol{\xi}, \boldsymbol{z}}$ with $N=7$ can also be given in a similar way as conducted in this paper.

It is also interesting to find an algorithm to compute all (real) self-dual spaces with prescribed ramification data. The solutions to the Bethe ansatz equations described in [9] can be used to find nontrivial examples of self-dual spaces. 
The paper is organized as follows. We start with the standard notation of Lie theory in Section 2 and computations of characters of a product of symmetric groups in Section 3. Then we recall notation and definitions for osculating Schubert calculus and self-dual spaces in Section 4. In Section 5 we recall the connections between Gaudin model of types B, C and self-dual spaces of polynomials. The symmetry of higher Gaudin Hamiltonians with respect to Shapovalov form and the key lemma from linear algebra are discussed in Section 6. In Section 7 we prove our main results, see Theorem 7.2 and Corollary 7.4. Finally, we display some simple data computed from Corollary 7.4 in Section 8.

\section{Simple Lie algebras}

Let $\mathfrak{g}$ be a simple Lie algebra over $\mathbb{C}$ with Cartan matrix $A=\left(a_{i, j}\right)_{i, j=1}^{r}$, where $r$ is the rank of $\mathfrak{g}$. Let $D=\operatorname{diag}\left(d_{1}, \ldots, d_{r}\right)$ be the diagonal matrix with positive relatively prime integers $d_{i}$ such that $D A$ is symmetric.

Let $\mathfrak{h} \subset \mathfrak{g}$ be a Cartan subalgebra with the Cartan decomposition $\mathfrak{g}=\mathfrak{n}_{-} \oplus \mathfrak{h} \oplus \mathfrak{n}_{+}$. Fix simple roots $\alpha_{1}, \ldots, \alpha_{r}$ in $\mathfrak{h}^{*}$. Let $\check{\alpha}_{1}, \ldots, \check{\alpha}_{r} \in \mathfrak{h}$ be the corresponding coroots. Fix a nondegenerate invariant bilinear form $($,$) on \mathfrak{g}$ such that $\left(\check{\alpha}_{i}, \check{\alpha}_{j}\right)=a_{i, j} / d_{j}$. The corresponding bilinear form on $\mathfrak{h}^{*}$ is given by $\left(\alpha_{i}, \alpha_{j}\right)=d_{i} a_{i, j}$. We have $\left\langle\lambda, \check{\alpha}_{i}\right\rangle=2\left(\lambda, \alpha_{i}\right) /\left(\alpha_{i}, \alpha_{i}\right)$ for $\lambda \in \mathfrak{h}^{*}$. In particular, $\left\langle\alpha_{j}, \check{\alpha}_{i}\right\rangle=a_{i, j}$. Let $\omega_{1}, \ldots, \omega_{r} \in \mathfrak{h}^{*}$ be the fundamental weights, $\left\langle\omega_{j}, \check{\alpha}_{i}\right\rangle=\delta_{i, j}$.

Let $\mathcal{P}=\left\{\lambda \in \mathfrak{h}^{*} \mid\left\langle\lambda, \check{\alpha}_{i}\right\rangle \in \mathbb{Z}, i=1, \ldots, r\right\}$ and $\mathcal{P}^{+}=\left\{\lambda \in \mathfrak{h}^{*} \mid\left\langle\lambda, \check{\alpha}_{i}\right\rangle \in \mathbb{Z}_{\geqslant 0}, i=1, \ldots, r\right\}$ be the weight lattice and the cone of dominant integral weights.

Let $e_{1}, \ldots, e_{r} \in \mathfrak{n}_{+}, \check{\alpha}_{1}, \ldots, \check{\alpha}_{r} \in \mathfrak{h}, f_{1}, \ldots, f_{r} \in \mathfrak{n}_{-}$be the Chevalley generators of $\mathfrak{g}$.

Given a $\mathfrak{g}$-module $M$, denote by $(M)^{\mathfrak{g}}$ the subspace of $\mathfrak{g}$-invariants in $M$. The subspace $(M)^{\mathfrak{g}}$ is the multiplicity space of the trivial $\mathfrak{g}$-module in $M$.

A sequence of nonnegative integers $\xi=\left(\xi_{1}, \ldots, \xi_{k}\right)$ such that $\xi_{1} \geqslant \xi_{2} \geqslant \cdots \geqslant \xi_{k} \geqslant 0$ is called a partition with at most $k$ parts. Set $|\xi|=\sum_{i=1}^{k} \xi_{i}$.

For $\lambda \in \mathfrak{h}^{*}$, let $V_{\lambda}$ be the irreducible $\mathfrak{g}$-module with highest weight $\lambda$. For any $\mathfrak{g}$-weights $\lambda$ and $\mu$, it is well known that $\operatorname{dim}\left(V_{\lambda} \otimes V_{\mu}\right)^{\mathfrak{g}}=\delta_{\lambda, \mu}$ for $\mathfrak{g}=\mathfrak{s o}_{2 r+1}, \mathfrak{s p}_{2 r}$.

For any Lie algebra $\mathfrak{g}$, denote by $\mathcal{U}(\mathfrak{g})$ the universal enveloping algebra of $\mathfrak{g}$.

\section{Characters of the symmetric groups}

Let $\mathfrak{g}_{N}$ be the Lie algebra $\mathfrak{s o}_{2 r+1}$ if $N=2 r$ or the Lie algebra $\mathfrak{s p}_{2 r}$ if $N=2 r+1, r \geqslant 2$. We also set $\mathfrak{g}_{3}=\mathfrak{s l}_{2}$. Let $\mathrm{G}_{N}$ be the respective classical group with Lie algebra $\mathfrak{g}_{N}$.

Let $\mathfrak{S}_{k}$ be the symmetric group permuting a set of $k$ elements. In this section we deduce a formula for characters of a product of the symmetric groups acting on a tensor product of finite-dimensional irreducible $\mathfrak{g}_{N}$-modules.

For each dominant integral $\mathfrak{g}_{N}$-weight $\lambda$, denote by $\bar{\lambda}=\left(\bar{\lambda}_{1}, \ldots, \bar{\lambda}_{r}\right)$ the partition with at most $r$ parts such that

$$
2\left\langle\lambda, \check{\alpha}_{i}\right\rangle=\bar{\lambda}_{i}-\bar{\lambda}_{i+1}, \quad i=1, \ldots, r-1, \quad \text { and } \quad \bar{\lambda}_{r}= \begin{cases}\left\langle\lambda, \check{\alpha}_{r}\right\rangle, & \text { if } N=2 r \\ 2\left\langle\lambda, \check{\alpha}_{r}\right\rangle, & \text { if } N=2 r+1 .\end{cases}
$$

Define an anti-symmetric Laurent polynomial $\Delta_{N}$ in $x_{1}, \ldots, x_{r}$ as follows

$$
\Delta_{N}=\operatorname{det}\left(x_{i}^{N+1-2 j}-x_{i}^{-(N+1-2 j)}\right)_{i, j=1}^{r} .
$$

We call $\Delta_{N}$ the Vandermonde determinant of $\mathfrak{g}_{N}$. 
Let $\lambda$ be a dominant integral $\mathfrak{g}_{N}$-weight. It is well known that the character of the module $V_{\lambda}$ is given by

$$
\mathcal{S}_{\lambda}^{N}\left(x_{1}, \ldots, x_{r}\right)=\operatorname{tr}_{V_{\lambda}} X_{N}=\frac{\operatorname{det}\left(x_{i}^{\bar{\lambda}_{j}+N+1-2 j}-x_{i}^{-\left(\bar{\lambda}_{j}+N+1-2 j\right)}\right)_{i, j=1}^{r}}{\Delta_{N}},
$$

where $X_{N} \in \mathrm{G}_{N}$ is given by

$$
X_{N}= \begin{cases}\operatorname{diag}\left(x_{1}^{2}, \ldots, x_{r}^{2}, 1, x_{r}^{-2}, \ldots, x_{1}^{-2}\right), & \text { if } N=2 r \\ \operatorname{diag}\left(x_{1}^{2}, \ldots, x_{r}^{2}, x_{r}^{-2}, \ldots, x_{1}^{-2}\right), & \text { if } N=2 r+1 .\end{cases}
$$

We call $\mathcal{S}_{\lambda}^{N}$ the Schur function of $\mathfrak{g}_{N}$ associated with the weight $\lambda$.

Note that $\mathcal{S}_{\lambda}^{N}$ are symmetric Laurent polynomials in $x_{1}, \ldots, x_{r}, \mathcal{S}_{\lambda}^{N} \in\left(\mathbb{C}\left[x_{1}^{ \pm 1}, \ldots, x_{r}^{ \pm 1}\right]\right)^{\mathfrak{S}_{r}}$.

Let $\lambda^{(1)}, \ldots, \lambda^{(s)}$ be a sequence of dominant integral $\mathfrak{g}_{N}$-weights and $k_{1}, \ldots, k_{s}$ a sequence of positive integers. Consider the tensor product of $\mathfrak{g}_{N}$-modules

$$
V_{\boldsymbol{\lambda}}=V_{\lambda^{(1)}}^{\otimes k_{1}} \otimes V_{\lambda^{(2)}}^{\otimes k_{2}} \otimes \cdots \otimes V_{\lambda^{(s)}}^{\otimes k_{s}}
$$

and its decomposition into irreducible $\mathfrak{g}_{N}$-submodules

$$
V_{\boldsymbol{\lambda}}=\bigoplus_{\mu} V_{\mu} \otimes M_{\boldsymbol{\lambda}, \mu}
$$

By permuting the corresponding tensor factors of $V_{\boldsymbol{\lambda}}$, the product of symmetric groups $\mathfrak{S}_{\boldsymbol{k}}=$ $\mathfrak{S}_{k_{1}} \times \mathfrak{S}_{k_{2}} \times \cdots \times \mathfrak{S}_{k_{s}}$ acts naturally on $V_{\boldsymbol{\lambda}}$. Note that the $\mathfrak{S}_{\boldsymbol{k}}$-action commutes with the $\mathfrak{g}_{N}$-action, therefore the group $\mathfrak{S}_{\boldsymbol{k}}$ acts on the multiplicity space $M_{\boldsymbol{\lambda}, \mu}$ for all $\mu$.

For $\sigma=\sigma_{1} \times \sigma_{2} \times \cdots \times \sigma_{s} \in \mathfrak{S}_{\boldsymbol{k}}, \sigma_{i} \in \mathfrak{S}_{k_{i}}$. Suppose all $\sigma_{i}$ are written as a product of disjoint cycles. Denote by $c_{i}$ the number of cycles in the product representing $\sigma_{i}$ and $l_{i j}, j=1, \ldots, c_{i}$, the lengths of cycles. Note that $l_{i, 1}+\cdots+l_{i, c_{i}}=k_{i}$.

We then consider the value of the character of $\mathfrak{S}_{\boldsymbol{k}}$ corresponding to the representation $M_{\boldsymbol{\lambda}, \mu}$ on $\sigma$. Let $\chi_{\boldsymbol{\lambda}, \mu}=\operatorname{tr}_{M_{\boldsymbol{\lambda}, \mu}}$.

Proposition 3.1. The character value $\chi_{\boldsymbol{\lambda}, \mu}(\sigma)$ equals the coefficient of the monomial

$$
x_{1}^{\bar{\mu}_{1}+N-1} x_{2}^{\bar{\mu}_{2}+N-3} \cdots x_{r}^{\bar{\mu}_{r}+N+1-2 r}
$$

in the Laurent polynomial

$$
\Delta_{N} \cdot \prod_{i=1}^{s} \prod_{j=1}^{c_{i}} \mathcal{S}_{\lambda^{(i)}}^{N}\left(x_{1}^{l_{i j}}, \ldots, x_{r}^{l_{i j}}\right)
$$

Proof. The proof of the statement is similar to that of [13, Proposition 2.1].

\section{Osculating Schubert calculus and self-dual spaces}

Let $N, d \in \mathbb{Z}_{>0}$ be such that $N \leqslant d$. Consider $\mathbb{P}^{1}:=\mathbb{C} \cup\{\infty\}$. Set

$$
\begin{aligned}
& \stackrel{\circ}{\mathbb{P}}_{n}:=\left\{\boldsymbol{z}=\left(z_{1}, \ldots, z_{n}\right) \in\left(\mathbb{P}^{1}\right)^{n} \mid z_{i} \neq z_{j} \text { for } 1 \leqslant i<j \leqslant n\right\}, \\
& \mathbb{R}^{\stackrel{\circ}{P}}:=\left\{\boldsymbol{z}=\left(z_{1}, \ldots, z_{n}\right) \in \stackrel{\circ}{\mathbb{P}}_{n} \mid z_{i} \in \mathbb{R} \text { or } z_{i}=\infty, \text { for } 1 \leqslant i \leqslant n\right\} .
\end{aligned}
$$




\subsection{Osculating Schubert calculus}

Let $\mathbb{C}_{d}[x]$ be the space of polynomials in $x$ with complex coefficients of degree less than $d$. We have $\operatorname{dim} \mathbb{C}_{d}[x]=d$. Let $\operatorname{Gr}(N, d)$ be the Grassmannian of all $N$-dimensional subspaces in $\mathbb{C}_{d}[x]$. The Grassmannian $\operatorname{Gr}(N, d)$ is a smooth projective complex variety of dimension $N(d-N)$.

Let $\mathbb{R}_{d}[x] \subset \mathbb{C}_{d}[x]$ be the set of polynomials in $x$ with real coefficients of degree less than $d$. Let $\operatorname{Gr}^{\mathbb{R}}(N, d) \subset \operatorname{Gr}(N, d)$ be the set of subspaces which have a basis consisting of polynomials with all coefficients real. For $X \in \operatorname{Gr}(N, d)$ we have $X \in \operatorname{Gr}^{\mathbb{R}}(N, d)$ if and only if $\operatorname{dim}_{\mathbb{R}}\left(X \cap \mathbb{R}_{d}[x]\right)=N$. We call such points $X$ real.

For a complete flag $\mathcal{F}=\left\{0 \subset \mathscr{F}_{1} \subset \mathscr{F}_{2} \subset \cdots \subset \mathscr{F}_{d}=\mathbb{C}_{d}[x]\right\}$ and a partition $\xi=\left(\xi_{1}, \ldots, \xi_{N}\right)$ such that $\xi_{1} \leqslant d-N$, the Schubert cell $\Omega_{\xi}(\mathcal{F}) \subset \operatorname{Gr}(N, d)$ is given by

$$
\begin{gathered}
\Omega_{\xi}(\mathcal{F})=\left\{X \in \operatorname{Gr}(N, d) \mid \operatorname{dim}\left(X \cap \mathscr{F}_{d-j-\xi_{N-j}}\right)=N-j,\right. \\
\left.\operatorname{dim}\left(X \cap \mathscr{F}_{d-j-\xi_{N-j}-1}\right)=N-j-1\right\} .
\end{gathered}
$$

Note that $\operatorname{codim} \Omega_{\xi}(\mathcal{F})=|\xi|$.

Let $\mathcal{F}(\infty)$ be the complete flag given by

$$
\mathcal{F}(\infty)=\left\{0 \subset \mathbb{C}_{1}[x] \subset \mathbb{C}_{2}[x] \subset \cdots \subset \mathbb{C}_{d}[x]\right\}
$$

The subspace $X$ is a point of $\Omega_{\xi}(\mathcal{F}(\infty))$ if and only if for every $i=1, \ldots, N$, it contains a polynomial of degree $d-i-\xi_{N+1-i}$.

For $z \in \mathbb{C}$, consider the complete flag

$$
\mathcal{F}(z)=\left\{0 \subset(x-z)^{d-1} \mathbb{C}_{1}[x] \subset(x-z)^{d-2} \mathbb{C}_{2}[x] \subset \cdots \subset \mathbb{C}_{d}[x]\right\} .
$$

The subspace $X$ is a point of $\Omega_{\xi}(\mathcal{F}(z))$ if and only if for every $i=1, \ldots, N$, it contains a polynomial with a root at $z$ of order exactly $\xi_{i}+N-i$.

A point $z \in \mathbb{C}$ is called a base point for a subspace $X \subset \mathbb{C}_{d}[x]$ if $\varphi(z)=0$ for every $\varphi \in X$.

Let $\boldsymbol{\xi}=\left(\xi^{(1)}, \ldots, \xi^{(n)}\right)$ be a sequence of partitions with at most $N$ parts and $\boldsymbol{z}=\left(z_{1}, \ldots, z_{n}\right) \in \stackrel{\circ}{\mathbb{P}}_{n}$. Set $|\boldsymbol{\xi}|=\sum_{s=1}^{n}\left|\xi^{(s)}\right|$.

Assuming $|\boldsymbol{\xi}|=N(d-N)$, denote by $\Omega_{\boldsymbol{\xi}, \boldsymbol{z}}$ the intersection of the Schubert cells

$$
\Omega_{\boldsymbol{\xi}, \boldsymbol{z}}=\bigcap_{s=1}^{n} \Omega_{\xi^{(s)}}\left(\mathcal{F}\left(z_{s}\right)\right) .
$$

Note that due to our assumption, $\Omega_{\boldsymbol{\xi}, \boldsymbol{z}}$ is a finite subset of $\operatorname{Gr}(N, d)$.

Define a sequence of polynomials $\boldsymbol{T}=\left(T_{1}, \ldots, T_{N}\right)$ by the formulas

$$
T_{i}(x)=\prod_{s=1}^{n}\left(x-z_{s}\right)^{\xi_{i}^{(s)}-\xi_{i+1}^{(s)}}, \quad i=1, \ldots, N
$$

where $\xi_{N+1}^{(s)}=0$. Here and in what follows we use the convention that $x-z_{s}$ is considered as the constant function 1 if $z_{s}=\infty$. We say that $\boldsymbol{T}$ is associated with $\boldsymbol{\xi}, \boldsymbol{z}$.

\subsection{Self-dual spaces}

Let $X \in \operatorname{Gr}(N, d)$ be an $N$-dimensional subspace of polynomials in $x$. Given a polynomial $\psi$ in $x$, denote by $\psi \cdot X$ the space of polynomials of the form $\psi \cdot \varphi$ for all $\varphi \in X$. 
Let $X^{\vee}$ be the $N$-dimensional space of polynomials which are Wronskian determinants of $N-1$ elements of $X$

$$
X^{\vee}=\left\{\operatorname{det}\left(d^{i-1} \varphi_{j} / d x^{i-1}\right)_{i, j=1}^{N-1}, \varphi_{j}(x) \in X\right\} .
$$

The space $X$ is called self-dual if $X^{\vee}=\psi \cdot X$ for some polynomial $\psi(x)$, see [16].

Let $\operatorname{sGr}(N, d)$ be the set of all self-dual spaces in $\operatorname{Gr}(N, d)$. We call $\operatorname{sGr}(N, d)$ the self-dual Grassmannian. The self-dual Grassmannian $\operatorname{sGr}(N, d)$ is an algebraic subset of $\operatorname{Gr}(N, d)$.

Denote by $\mathrm{s} \Omega_{\boldsymbol{\xi}, \boldsymbol{z}}$ the set of all self-dual spaces in $\Omega_{\boldsymbol{\xi}, \boldsymbol{z}}$

$$
\mathrm{s} \Omega_{\boldsymbol{\xi}, \boldsymbol{z}}=\Omega_{\boldsymbol{\xi}, \boldsymbol{z}} \bigcap \mathrm{sGr}(N, d) .
$$

Let $\mu$ be a dominant integral $\mathfrak{g}_{N}$-weight and $k \in \mathbb{Z}_{\geqslant 0}$. Define a partition $\mu_{A, k}$ with at most $N$ parts by the rule: $\left(\mu_{A, k}\right)_{N}=k$ and

$$
\left(\mu_{A, k}\right)_{i}-\left(\mu_{A, k}\right)_{i+1}= \begin{cases}\left\langle\mu, \check{\alpha}_{i}\right\rangle, & \text { if } 1 \leqslant i \leqslant\left[\frac{N}{2}\right] \\ \left\langle\mu, \check{\alpha}_{N-i}\right\rangle, & \text { if }\left[\frac{N}{2}\right]<i \leqslant N-1 .\end{cases}
$$

We call $\mu_{A, k}$ the partition associated with weight $\mu$ and integer $k$.

Let $\boldsymbol{\lambda}=\left(\lambda^{(1)}, \ldots, \lambda^{(n)}\right)$ be a sequence of dominant integral $\mathfrak{g}_{N^{-w e i g h t s}}$ and let $\boldsymbol{k}=\left(k_{1}, \ldots, k_{n}\right)$ be an $n$-tuple of nonnegative integers. Then denote $\boldsymbol{\lambda}_{A, \boldsymbol{k}}=\left(\lambda_{A, k_{1}}^{(1)}, \ldots, \lambda_{A, k_{n}}^{(n)}\right)$ the sequence of partitions associated with $\lambda^{(s)}$ and $k_{s}, s=1, \ldots, n$.

We use the notation $\mu_{A}=\mu_{A, 0}$ and $\boldsymbol{\lambda}_{A}=\boldsymbol{\lambda}_{A,(0, \ldots, 0)}$.

Lemma 4.1 ([10]). If $\boldsymbol{\xi}$ is a sequence of partitions with at most $N$ parts such that $|\boldsymbol{\xi}|=N(d-N)$ and $\mathrm{s} \Omega_{\boldsymbol{\xi}, \boldsymbol{z}}$ is nonempty, then $\boldsymbol{\xi}$ has the form $\boldsymbol{\xi}=\boldsymbol{\lambda}_{A, \boldsymbol{k}}$ for a sequence of dominant integral $\mathfrak{g}_{N^{-}}$ weights $\boldsymbol{\lambda}=\left(\lambda^{(1)}, \ldots, \lambda^{(n)}\right)$ and a sequence of nonnegative integers $\boldsymbol{k}=\left(k_{1}, \ldots, k_{n}\right)$. The pair $(\boldsymbol{\lambda}, \boldsymbol{k})$ is uniquely determined by $\boldsymbol{\xi}$.

In what follows we write $\Omega_{\boldsymbol{\lambda}, \boldsymbol{z}}, \Omega_{\boldsymbol{\lambda}, \boldsymbol{k}, \boldsymbol{z}}, \mathrm{s} \Omega_{\boldsymbol{\lambda}, \boldsymbol{z}}, \mathrm{s} \Omega_{\boldsymbol{\lambda}, \boldsymbol{k}, \boldsymbol{z}}$ for $\Omega_{\boldsymbol{\lambda}_{A}, \boldsymbol{z}}, \Omega_{\boldsymbol{\lambda}_{A, \boldsymbol{k}}, \boldsymbol{z}}, \mathrm{s} \Omega_{\boldsymbol{\lambda}_{A}, \boldsymbol{z}}, \mathrm{s} \Omega_{\boldsymbol{\lambda}_{A, \boldsymbol{k}}, \boldsymbol{z}}$, respectively.

Note that $\left|\boldsymbol{\lambda}_{A, \boldsymbol{k}}\right|=\left|\boldsymbol{\lambda}_{A}\right|+N|\boldsymbol{k}|$, where $|\boldsymbol{k}|=k_{1}+\cdots+k_{n}$. Suppose $\left|\boldsymbol{\lambda}_{A}\right|=N(d-N)$, there exists a bijection between $\Omega_{\boldsymbol{\lambda}, \boldsymbol{z}}$ in $\operatorname{Gr}(N, d)$ and $\Omega_{\boldsymbol{\lambda}, \boldsymbol{k}, \boldsymbol{z}}$ in $\operatorname{Gr}(N, d+|\boldsymbol{k}|)$ given by

$$
\Omega_{\boldsymbol{\lambda}, \boldsymbol{z}} \rightarrow \Omega_{\boldsymbol{\lambda}, \boldsymbol{k}, \boldsymbol{z}}, \quad X \mapsto \prod_{s=1}^{n}\left(x-z_{s}\right)^{k_{s}} \cdot X .
$$

Moreover, (4.5) restricts to a bijection between $\mathrm{s} \Omega_{\boldsymbol{\lambda}, \boldsymbol{z}}$ in $\operatorname{sGr}(N, d)$ and $\mathrm{s} \Omega_{\boldsymbol{\lambda}, \boldsymbol{k}, \boldsymbol{z}}$ in $\mathrm{s} \operatorname{Gr}(N, d+|\boldsymbol{k}|)$.

\section{Gaudin model}

Let $\mathfrak{g}[t]=\mathfrak{g} \otimes \mathbb{C}[t]$ be the Lie algebra of $\mathfrak{g}$-valued polynomials with the pointwise commutator. We call it the current algebra of $\mathfrak{g}$. We identify the Lie algebra $\mathfrak{g}$ with the subalgebra $\mathfrak{g} \otimes 1$ of constant polynomials in $\mathfrak{g}[t]$.

It is convenient to collect elements of $\mathfrak{g}[t]$ in generating series of a formal variable $x$. For $g \in \mathfrak{g}$, set

$$
g(x)=\sum_{k=0}^{\infty}\left(g \otimes t^{k}\right) x^{-k-1} .
$$

For each $a \in \mathbb{C}$, we have the evaluation homomorphism ev $a \mathfrak{g}[t] \rightarrow \mathfrak{g}$ where $\mathrm{ev}_{a}$ sends $g \otimes t^{s}$ to $a^{s} g$ for all $g \in \mathfrak{g}$ and $s \in \mathbb{Z}_{\geqslant 0}$. Its restriction to the subalgebra $\mathfrak{g} \subset \mathfrak{g}[t]$ is the identity map. 
For any $\mathfrak{g}$-module $M$, we denote by $M(a)$ the $\mathfrak{g}[t]$-module, obtained by pulling $M$ back through the evaluation homomorphism $\mathrm{ev}_{a}$. The $\mathfrak{g}[t]$-module $M(a)$ is called an evaluation module. The generating series $g(x)$ acts on the evaluation module $M(a)$ by $g /(x-a)$.

The Bethe algebra $\mathcal{B}$ (the algebra of higher Gaudin Hamiltonians) for a simple Lie algebra $\mathfrak{g}$ was described in [3]. The Bethe algebra $\mathcal{B}$ is a commutative subalgebra of $\mathcal{U}(\mathfrak{g}[t])$ which commutes with the subalgebra $\mathcal{U}(\mathfrak{g}) \subset \mathcal{U}(\mathfrak{g}[t])$. An explicit set of generators of the Bethe algebra in Lie algebras of types B, C, and D was given in [11].

Proposition 5.1 ([3, 11]). Let $N>3$. There exist elements $F_{i j} \in \mathfrak{g}_{N}, i, j=1, \ldots, N$, and polynomials $B_{s}(x)$ in $d^{k} F_{i j}(x) / d x^{k}, s=1, \ldots, N, k=0, \ldots, N$, such that the Bethe algebra $\mathcal{B}$ of $\mathfrak{g}_{N}$ is generated by the coefficients of $B_{s}(x)$ considered as formal power series in $x^{-1}$.

We denote $M(\infty)$ the $\mathfrak{g}_{N}$-module $M$ with the trivial action of the Bethe algebra $\mathcal{B}$, see [10] for more detail.

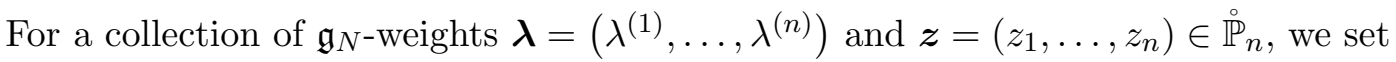

$$
V_{\boldsymbol{\lambda}, \boldsymbol{z}}=\bigotimes_{s=1}^{n} V_{\lambda^{(s)}}\left(z_{s}\right)
$$

considered as a $\mathcal{B}$-module. We also denote $V_{\boldsymbol{\lambda}}$ the module $V_{\boldsymbol{\lambda}, \boldsymbol{z}}$ considered as a $\mathfrak{g}_{N}$-module.

Let $\partial_{x}$ be the differentiation with respect to $x$. Define a formal differential operator

$$
\mathcal{D}^{\mathcal{B}}=\partial_{x}^{N}+\sum_{i=1}^{N} B_{i}(x) \partial_{x}^{N-i}
$$

where

$$
B_{i}(x)=\sum_{j=i}^{\infty} B_{i j} x^{-j}
$$

and $B_{i j} \in \mathcal{U}\left(\mathfrak{g}_{N}[t]\right), j \in \mathbb{Z}_{\geqslant i}, i=1, \ldots, N$. The operator $\mathcal{D}^{\mathcal{B}}$ is called the universal operator.

Let $\boldsymbol{z}=\left(z_{1}, \ldots, z_{n}\right) \in \stackrel{\circ}{\mathbb{P}}_{n}$ and let $\boldsymbol{\lambda}=\left(\lambda^{(1)}, \ldots, \lambda^{(n)}\right)$ be a sequence of dominant integral $\mathfrak{g}_{N}$-weights. For every $g \in \mathfrak{g}_{N}$, the series $g(x)$ acts on $V_{\boldsymbol{\lambda}, \boldsymbol{z}}$ as a rational function of $x$.

Since the Bethe algebra $\mathcal{B}$ commutes with $\mathfrak{g}_{N}, \mathcal{B}$ acts on the invariant space $\left(V_{\boldsymbol{\lambda}, \boldsymbol{z}}\right)^{\mathfrak{g}_{N}}$. For $b \in \mathcal{B}$, denote by $b(\boldsymbol{\lambda}, \boldsymbol{z}) \in \operatorname{End}\left(\left(V_{\boldsymbol{\lambda}, \boldsymbol{z}}\right)^{\mathfrak{g}_{N}}\right)$ the corresponding linear operator.

Given a common eigenvector $v \in\left(V_{\boldsymbol{\lambda}, \boldsymbol{z}}\right)^{\mathfrak{g}_{N}}$ of the operators $b(\boldsymbol{\lambda}, \boldsymbol{z})$, denote by $b(\boldsymbol{\lambda}, \boldsymbol{z} ; v)$ the corresponding eigenvalues, and define the scalar differential operator

$$
\mathcal{D}_{v}=\partial_{x}^{N}+\sum_{i=1}^{N} \sum_{j=i}^{\infty} B_{i j}(\boldsymbol{\lambda}, \boldsymbol{z} ; v) x^{-j} \partial_{x}^{N-i} .
$$

The following theorem connects self-dual spaces in the $\operatorname{Grassmannian} \operatorname{Gr}(N, d)$ with the Gaudin model associated to $\mathfrak{g}_{N}$.

Theorem 5.2 ([10]). Let $N>3$. There exists a choice of generators $B_{i}(x)$ of the Bethe algebra $\mathcal{B}$, such that for any sequence of dominant integral $\mathfrak{g}_{N}$-weights $\boldsymbol{\lambda}=\left(\lambda^{(1)}, \ldots, \lambda^{(n)}\right)$, any $\boldsymbol{z} \in \stackrel{\circ}{\mathbb{P}}_{n}$, and any $\mathcal{B}$-eigenvector $v \in\left(V_{\boldsymbol{\lambda}, \boldsymbol{z}}\right)^{\mathfrak{g}_{N}}$, we have

$$
\operatorname{Ker}\left(\left(T_{1} \cdots T_{N}\right)^{1 / 2} \cdot \mathcal{D}_{v} \cdot\left(T_{1} \cdots T_{N}\right)^{-1 / 2}\right) \in \mathrm{s} \Omega_{\boldsymbol{\lambda}, \boldsymbol{z}},
$$

where $\boldsymbol{T}=\left(T_{1}, \ldots, T_{N}\right)$ is associated with $\boldsymbol{\lambda}_{A}, \boldsymbol{z}$.

Moreover, if $\left|\boldsymbol{\lambda}_{A}\right|=N(d-N)$, then this defines a bijection between the joint eigenvalues of $\mathcal{B}$ on $\left(V_{\boldsymbol{\lambda}, \boldsymbol{z}}\right)^{\mathfrak{g}_{N}}$ and $\mathrm{s} \Omega_{\boldsymbol{\lambda}, \boldsymbol{z}} \subset \operatorname{Gr}(N, d)$. 


\section{Shapovalov form and the key lemma}

\subsection{Shapovalov form}

Define the anti-involution $\varpi: \mathfrak{g}_{N} \rightarrow \mathfrak{g}_{N}$ sending $e_{1}, \ldots, e_{r}, \check{\alpha}_{1}, \ldots, \check{\alpha}_{r}, f_{1}, \ldots, f_{r}$ to $f_{1}, \ldots, f_{r}$, $\check{\alpha}_{1}, \ldots, \check{\alpha}_{r}, e_{1}, \ldots, e_{r}$, respectively.

For any dominant integral $\mathfrak{g}_{N}$-weight $\lambda$, the irreducible $\mathfrak{g}_{N}$-module $V_{\lambda}$ admits a positive definite Hermitian form $(\cdot, \cdot)_{\lambda}$ such that $(g v, w)_{\lambda}=(v, \overline{\varpi(g)} w)_{\lambda}$ for any $v, w \in V_{\lambda}$ and $g \in \mathfrak{g}_{N}$. Such a form is unique up to multiplication by a positive real number. We call this form the Shapovalov form.

Let $\boldsymbol{\lambda}=\left(\lambda^{(1)}, \ldots, \lambda^{(n)}\right)$ be a sequence of dominant integral $\mathfrak{g}_{N}$-weights. We define the positive definite Hermitian form $(\cdot, \cdot)_{\boldsymbol{\lambda}}$ on the tensor product $V_{\boldsymbol{\lambda}}$ as the product of Shapovalov forms on the tensor factors. The form $(\cdot, \cdot)_{\boldsymbol{\lambda}}$ induces a positive definite Hermitian form $(\cdot \mid \cdot)_{\boldsymbol{\lambda}}$ on $\left(V_{\boldsymbol{\lambda}, \boldsymbol{z}}\right)^{\mathfrak{g}_{N}}$.

Proposition 6.1. For any $i=1, \ldots, N, j \in \mathbb{Z}_{\geqslant i}$, and any $v, w \in\left(V_{\boldsymbol{\lambda}, \boldsymbol{z}}\right)^{\mathfrak{g}_{N}}$, we have

$$
\left(B_{i j}(\boldsymbol{\lambda}, \boldsymbol{z}) v \mid w\right)_{\boldsymbol{\lambda}}=\left(v \mid B_{i j}(\boldsymbol{\lambda}, \overline{\boldsymbol{z}}) w\right)_{\boldsymbol{\lambda}},
$$

where $B_{i j}$ are given by $(5.2), \bar{z}=\left(\bar{z}_{1}, \ldots, \bar{z}_{n}\right)$ and the bar stands for the complex conjugation.

Proof. We prove the proposition in Section 6.3.

If $\boldsymbol{z} \in \mathbb{R} \stackrel{\circ}{\mathbb{P}}_{n}$, then $B_{i j}(\boldsymbol{\lambda}, \boldsymbol{z})$ are self-adjoint with respect to the Shapovalov form. Therefore all $B_{i j}(\boldsymbol{\lambda}, \boldsymbol{z})$ are simultaneously diagonalizable and all eigenvalues of $B_{i j}(\boldsymbol{\lambda}, \boldsymbol{z})$ are real.

The following statement is also known.

Theorem 6.2 ([18]). For generic $\boldsymbol{z} \in \stackrel{\circ}{\mathbb{P}}_{n}$, the action of the Bethe algebra $\mathcal{B}$ on $\left(V_{\boldsymbol{\lambda}, \boldsymbol{z}}\right)^{\mathfrak{g}_{N}}$ is diagonalizable and has simple spectrum. In particular, this statement holds for any sequence $z \in \mathbb{R}^{\mathbb{P}}{ }_{n}$.

If some of the partitions $\lambda^{(1)}, \ldots, \lambda^{(n)}$ coincide, the operators $b(\boldsymbol{\lambda}, \boldsymbol{z})$ admit additional symmetry. Assume that $\lambda^{(i)}=\lambda^{(i+1)}$ for some $i$. Let $P_{i} \in \operatorname{End}\left(V_{\boldsymbol{\lambda}}\right)$ be the flip of the $i$-th and $(i+1)$-st tensor factors and $\tilde{\boldsymbol{z}}^{(i)}=\left(z_{1}, \ldots, z_{i-1}, z_{i+1}, z_{i}, z_{i+2}, \ldots, z_{n}\right)$.

Lemma 6.3. For any $b \in \mathcal{B}$, we have $P_{i} b(\boldsymbol{\lambda}, \boldsymbol{z}) P_{i}=b\left(\boldsymbol{\lambda}, \tilde{\boldsymbol{z}}^{(i)}\right)$.

\subsection{Self-adjoint operators with respect to indefinite Hermitian form}

In this section we recall the key lemma from linear algebra, see [17].

Given a finite-dimensional vector space $M$, a linear operator $\mathfrak{T} \in \operatorname{End}(M)$, and a number $\alpha \in \mathbb{C}$, let $M_{\mathfrak{T}}(\alpha)=\operatorname{ker}(\mathfrak{T}-\alpha)^{\operatorname{dim} M}$. When $M_{\mathfrak{T}}(\alpha)$ is not trivial, it is the subspace of generalized eigenvectors of $\mathfrak{T}$ with eigenvalue $\alpha$.

Lemma 6.4 ([17]). Let $M$ be a complex finite-dimensional vector space with a nondegenerate Hermitian form of signature $\kappa$, and let $\mathcal{A} \subset \operatorname{End}(M)$ be a commutative subalgebra over $\mathbb{R}$, whose elements are self-adjoint operators. Let $R=\bigcap_{\mathfrak{T} \in \mathcal{A}} \bigoplus_{\alpha \in \mathbb{R}} M_{\mathfrak{T}}(\alpha)$. Then the restriction of the Hermitian form on $R$ is nondegenerate and has signature $\kappa$. In particular, $\operatorname{dim} R \geqslant|\kappa|$.

\subsection{Proof of Proposition 6.1}

In this section, we give the proof of Proposition 6.1. We follow the convention of [12]. We only introduce the necessary notation and refer the reader to [11, Section 5] and [12, Section 3] for more detail. 
Proof of Proposition 6.1. We prove it for the case $N=2 r$ first.

Let $E_{i j}$ with $i, j=1, \ldots, 2 r+1$ be the standard basis of $\mathfrak{g l}_{2 r+1}$. The Lie subalgebra of $\mathfrak{g l}_{2 r+1}$ generated by the elements $F_{i j}=E_{i j}-E_{2 r+2-j, 2 r+2-i}$ is isomorphic to the Lie algebra $\mathfrak{s o}_{2 r+1}=\mathfrak{g}_{N}$. With this isomorphism, the anti-involution $\varpi: \mathfrak{g}_{N} \rightarrow \mathfrak{g}_{N}$ is realized by taking transposition, $F_{i j} \mapsto F_{j i}$. To be consistent with the notation in [12], we write $\mathfrak{g}$ for $\mathfrak{g}_{N}$. The number $N$ in [12] is $2 r+1$ in our notation.

We write $F_{i j}[s]$ for $F_{i j} \otimes t^{s}$ in the loop algebra $\mathfrak{g}\left[t, t^{-1}\right]$. Consider the affine Lie algebra $\widehat{\mathfrak{g}}=\mathfrak{g}\left[t, t^{-1}\right] \oplus \mathbb{C} K$, which is the central extension of the loop algebra $\mathfrak{g}\left[t, t^{-1}\right]$, where the element $K$ is central in $\widehat{\mathfrak{g}}$ and

$$
\left[g_{1}[k], g_{2}[l]\right]=\left[g_{1}, g_{2}\right][k+l]+k \delta_{k,-l}\left(g_{1}, g_{2}\right) K, \quad g_{1}, g_{2} \in \mathfrak{g}, \quad k, l \in \mathbb{Z} .
$$

Consider the extended affine Lie algebra $\widehat{\mathfrak{g}} \oplus \mathbb{C} \tau=\mathfrak{g}\left[t, t^{-1}\right] \oplus \mathbb{C} K \oplus \mathbb{C} \tau$, where $\tau$ satisfies

$$
\left[\tau, F_{i j}[s]\right]=-s F_{i j}[s-1], \quad[\tau, K]=0, \quad s \in \mathbb{Z} .
$$

Set $\mathcal{U}=\mathcal{U}(\widehat{\mathfrak{g}} \oplus \mathbb{C} \tau)$ and fix $m \in\{1, \ldots, N\}$. Introduce the element $F[s]_{a}$ of the algebra $\left(\text { End }\left(\mathbb{C}^{2 r+1}\right)\right)^{\otimes m} \otimes \mathcal{U}$, see $[12$, equation (3.5)], by

$$
F[s]_{a}=\sum_{i, j=1}^{2 r+1} 1^{\otimes(a-1)} \otimes e_{i j} \otimes 1^{\otimes(m-a)} \otimes F_{i j}[s],
$$

where $e_{i j} \in$ End $\left(\mathbb{C}^{2 r+1}\right)$ denote the standard matrix units. The map $\varpi$ induces an antiinvolution

$$
\varpi: \mathcal{U}\left(t^{-1} \mathfrak{g}\left[t^{-1}\right]\right) \rightarrow \mathcal{U}\left(t^{-1} \mathfrak{g}\left[t^{-1}\right]\right), \quad F_{i j}[s] \mapsto F_{j i}[s], \quad s \in \mathbb{Z}_{\leqslant-1} .
$$

For $1 \leqslant a<b \leqslant m$, consider the operators $P_{a b}$ and $Q_{a b}$ in $\left(\text { End }\left(\mathbb{C}^{2 r+1}\right)\right)^{\otimes m}$ defined as follows

$$
\begin{aligned}
P_{a b} & =\sum_{i, j=1}^{2 r+1} 1^{\otimes(a-1)} \otimes e_{i j} \otimes 1^{\otimes(b-a-1)} \otimes e_{j i} \otimes 1^{\otimes(m-b)}, \\
Q_{a b} & =\sum_{i, j=1}^{2 r+1} 1^{\otimes(a-1)} \otimes e_{i j} \otimes 1^{\otimes(b-a-1)} \otimes e_{2 r+2-i, 2 r+2-j} \otimes 1^{\otimes(m-b)} .
\end{aligned}
$$

Set

$$
S^{(m)}=\frac{1}{m !} \prod_{1 \leqslant a<b \leqslant m}\left(1+\frac{P_{a b}}{b-a}-\frac{2 Q_{a b}}{2 r+2 b-2 a-1}\right),
$$

where the product is taken in the lexicographic order on the pairs $(a, b)$. The element $S^{(m)}$ is the symmetrizer of the Brauer algebra acting on $\left(\mathbb{C}^{2 r+1}\right)^{\otimes m}$. In particular, for any $1 \leqslant a<b \leqslant m$, the operator $S^{(m)}$ satisfies

$$
S^{(m)} Q_{a b}=Q_{a b} S^{(m)}=0, \quad S^{(m)} P_{a b}=P_{a b} S^{(m)}=S^{(m)} .
$$

Replacing $\tau$ with $\partial_{x}$ and $F_{i j}[-\ell-1]$ with $-\partial_{x}^{\ell} F_{i j}(x) / \ell$ !, where $F_{i j}(x)$ is defined in (5.1), for $\ell \in \mathbb{Z}_{\geqslant 0}$, in the element

$$
\frac{2 r+m-1}{2 r+2 m-1} \operatorname{tr} S^{(m)}\left(\tau+F[-1]_{1}\right) \cdots\left(\tau+F[-1]_{m}\right)
$$


see $[12$, formula $(3.26)]$, where the trace is taken on all $m$ copies of End $\left(\mathbb{C}^{2 r+1}\right)$, we get a differential operator

$$
\vartheta_{m 0}(x) \partial_{x}^{m}+\vartheta_{m 1}(x) \partial_{x}^{m-1}+\cdots+\vartheta_{m m}(x),
$$

where $\vartheta_{m i}(x)$ is a formal power series in $x^{-1}$ with coefficients in $\mathcal{U}(\mathfrak{g}[t])$. The Bethe subalgebra $\mathcal{B}$ of $\mathcal{U}(\mathfrak{g}[t])$ is generated by the coefficients of $\vartheta_{m i}(x), m=1, \ldots, N, i=0, \ldots, m$, see [11, Section 5].

Therefore, to prove the proposition, it suffices to show that the element

$$
\frac{2 r+m-1}{2 r+2 m-1} \operatorname{tr} S^{(m)}\left(\tau+F[-1]_{1}\right) \cdots\left(\tau+F[-1]_{m}\right)
$$

is stable under the anti-involution $\varpi$. Here $\varpi$ maps $\tau$ to $\tau$.

Applying transposition on $a$-th and $b$-th components to the commutator relation

$$
F[k]_{a} F[l]_{b}-F[l]_{b} F[k]_{a}=\left(P_{a b}-Q_{a b}\right) F[k+l]_{b}-F[k+l]_{b}\left(P_{a b}-Q_{a b}\right),
$$

see the proof of [12, Lemma 3.6], we get

$$
F^{\top}[k]_{a} F^{\top}[l]_{b}-F^{\top}[l]_{b} F^{\top}[k]_{a}=F^{\top}[k+l]_{b}\left(P_{a b}-Q_{a b}\right)-\left(P_{a b}-Q_{a b}\right) F^{\top}[k+l]_{b},
$$

for all $1 \leqslant a<b \leqslant m$. Here $\top$ stands for transpose, explicitly,

$$
F^{\top}[s]_{a}=\sum_{i, j=1}^{2 r+1} 1^{\otimes(a-1)} \otimes e_{i j} \otimes 1^{\otimes(m-a)} \otimes F_{j i}[s] .
$$

Thus one can use the same argument as in the proof of [12, Lemma 3.2] to show that the image of (6.1) under the anti-involution $\varpi$ equals

$$
\frac{2 r+m-1}{2 r+2 m-1} \operatorname{tr} S^{(m)}\left(\tau+F^{\top}[-1]_{1}\right) \cdots\left(\tau+F^{\top}[-1]_{m}\right) .
$$

By applying the simultaneous transposition $e_{i j} \rightarrow e_{j i}$ to all $m$ copies of End $\left(\mathbb{C}^{2 r+1}\right)$ we conclude that (6.2) coincides with (6.1) because the transformation takes each factor $\tau+F^{\top}[-1]_{a}$ to $\tau+F[-1]_{a}$ whereas the symmetrizer $S^{(m)}$ stays invariant. Hence we complete the proof of Proposition 6.1 for the case $N=2 r$.

The case $N=2 r+1$ is proved similarly, see for example [12, Lemma 3.9].

\section{The lower bound}

In this section we prove our main results - the lower bound for the number of real self-dual spaces in $\Omega_{\boldsymbol{\lambda}, \boldsymbol{z}}$, see Theorem 7.2 and Corollary 7.4 .

Recall the notation from Section 4. For positive integers $N, d$ such that $d \geqslant N$ we consider the Grassmannian $\operatorname{Gr}(N, d)$ of $N$-dimensional planes in the space $\mathbb{C}_{d}[x]$ of polynomials of degree less than $d$. A point $X \in \operatorname{Gr}(N, d)$ is called real if it has a basis consisting of polynomials with all coefficients real.

\subsection{The general case $N \geqslant 4$}

Let us first consider the case $N \geqslant 4$.

Let $\boldsymbol{\lambda}=\left(\lambda^{(1)}, \ldots, \lambda^{(n)}\right)$ be a sequence of dominant integral $\mathfrak{g}_{N^{-w e i g h t s}} \boldsymbol{k}=\left(k_{1}, \ldots, k_{n}\right)$ an $n$-tuple of nonnegative integers, and $\boldsymbol{z}=\left(z_{1}, \ldots, z_{n}\right) \in \stackrel{\circ}{\mathbb{P}}_{n}$. Suppose that $\left|\boldsymbol{\lambda}_{A, \boldsymbol{k}}\right|=N(d-N)$. Denote by $d(\boldsymbol{\lambda}, \boldsymbol{k}, \boldsymbol{z})$ the number of real points counted with multiplicities in $\mathrm{s} \Omega_{\boldsymbol{\lambda}, \boldsymbol{k}, \boldsymbol{z}} \subset \operatorname{Gr}(N, d)$. 
Clearly, $d(\boldsymbol{\lambda}, \boldsymbol{k}, \boldsymbol{z})=0$ unless the set $\left\{z_{1}, \ldots, z_{n}\right\}$ is invariant under the complex conjugation and $\left(\lambda^{(i)}, k_{i}\right)=\left(\lambda^{(j)}, k_{j}\right)$ whenever $z_{i}=\bar{z}_{j}$. In particular, the polynomial $\prod_{s=1}^{n}\left(x-z_{s}\right)^{k_{s}}$ has only real coefficients. It follows from (4.5) that the number of real points in $\mathrm{s} \Omega_{\boldsymbol{\lambda}, \boldsymbol{k}, \boldsymbol{z}} \subset \operatorname{Gr}(N, d)$ is equal to that of $\mathrm{s} \Omega_{\boldsymbol{\lambda}, \boldsymbol{z}} \subset \operatorname{Gr}(N, d-|\boldsymbol{k}|)$. From now on, we shall only consider the case that $\boldsymbol{k}=(0, \ldots, 0)$. We simply write $d(\boldsymbol{\lambda}, \boldsymbol{z})$ for $d(\boldsymbol{\lambda}, \boldsymbol{k}, \boldsymbol{z})$ if $\boldsymbol{k}=(0, \ldots, 0)$.

Let $\boldsymbol{T}=\left(T_{1}, \ldots, T_{N}\right)$ be associated with $\boldsymbol{\lambda}_{A, \boldsymbol{k}}, \boldsymbol{z}$. Note that if $\boldsymbol{z}, \boldsymbol{\lambda}, \boldsymbol{k}$ is invariant under conjugation, then the polynomial $T_{1} \cdots T_{N}$ also has only real coefficients.

In what follows we denote by $c$ the number of complex conjugate pairs in the set $\left\{z_{1}, \ldots, z_{n}\right\}$ and without loss of generality assume that $z_{1}=\bar{z}_{2}, \ldots, z_{2 c-1}=\bar{z}_{2 c}$ while $z_{2 c+1}, \ldots, z_{n}$ are real (one of them can be infinity). We will also always assume that $\lambda^{(1)}=\lambda^{(2)}, \ldots, \lambda^{(2 c-1)}=\lambda^{(2 c)}$.

Recall that for any $\boldsymbol{\lambda}$ and generic $\boldsymbol{z} \in \stackrel{\circ}{\mathbb{P}}_{n}$, all points of $\Omega_{\boldsymbol{\lambda}, \boldsymbol{z}}$ are multiplicity-free. The same also holds true with $\boldsymbol{\lambda}$ imposed above for any $c$.

Consider the decomposition of $V_{\boldsymbol{\lambda}}$ into irreducible $\mathfrak{g}_{N}$-submodules

$$
V_{\boldsymbol{\lambda}}=\bigoplus_{\mu} V_{\mu} \otimes M_{\boldsymbol{\lambda}, \mu}
$$

Then $M_{\boldsymbol{\lambda}, 0}=\left(V_{\boldsymbol{\lambda}}\right)^{\mathfrak{g}_{N}}$. Since $\lambda^{(2 i-1)}=\lambda^{(2 i)}$ for $i=1, \ldots, c$, the flip $P_{2 i-1}$ of the $(2 i-1)$-st and $2 i$-th tensor factors of $V_{\boldsymbol{\lambda}}$ commutes with the $\mathfrak{g}_{N}$-action and thus acts on $\left(V_{\boldsymbol{\lambda}}\right)^{\mathfrak{g}_{N}}$. Denote by $P_{\boldsymbol{\lambda}, c} \in \operatorname{End}\left(\left(V_{\boldsymbol{\lambda}}\right)^{\mathfrak{g}_{N}}\right)$ the action of the product $P_{1} P_{3} \cdots P_{2 c-1}$ on $\left(V_{\boldsymbol{\lambda}}\right)^{\mathfrak{g}_{N}}$.

The operator $P_{\boldsymbol{\lambda}, c}$ is self-adjoint relative to the Hermitian form $(\cdot \mid \cdot)_{\boldsymbol{\lambda}}$ on $\left(V_{\boldsymbol{\lambda}}\right)^{\mathfrak{g}_{N}}$ given in Section 6. Define a new Hermitian form $(\cdot, \cdot)_{\boldsymbol{\lambda}, c}$ on $\left(V_{\boldsymbol{\lambda}}\right)^{\mathfrak{g}_{N}}$ by the rule: for any $v, w \in\left(V_{\boldsymbol{\lambda}}\right)^{\mathfrak{g}_{N}}$

$$
(v, w)_{\boldsymbol{\lambda}, c}=\left(P_{\boldsymbol{\lambda}, c} v \mid w\right)_{\boldsymbol{\lambda}} .
$$

Denote by $q(\boldsymbol{\lambda}, c)$ the signature of the form $(\cdot, \cdot)_{\boldsymbol{\lambda}, c}$.

Proposition 7.1. The signature $q(\boldsymbol{\lambda}, c)$ equals the coefficient of the monomial

$$
x_{1}^{N-1} x_{2}^{N-3} \cdots x_{r}^{N+1-2 r},
$$

in the Laurent polynomial

$$
\Delta_{N} \cdot \prod_{i=1}^{c} \mathcal{S}_{\lambda^{(2 i)}}^{N}\left(x_{1}^{2}, \ldots, x_{r}^{2}\right) \prod_{j=2 c+1}^{n} \mathcal{S}_{\lambda^{(j)}}^{N}\left(x_{1}, \ldots, x_{r}\right) .
$$

Here $\Delta_{N}$ and $\mathcal{S}_{\lambda^{(s)}}^{N}$ are given by (3.1) and (3.2), respectively.

Proof. Since $P_{\boldsymbol{\lambda}, c}^{2}=1$ and $M_{\boldsymbol{\lambda}, 0}=\left(V_{\boldsymbol{\lambda}}\right)^{\mathfrak{g}_{N}}$, we have $q(\boldsymbol{\lambda}, c)=\operatorname{tr}_{M_{\boldsymbol{\lambda}, 0}} P_{\boldsymbol{\lambda}, c}$, and the claim follows from Proposition 3.1.

Theorem 7.2. The number $d(\boldsymbol{\lambda}, \boldsymbol{z})$ of real self-dual spaces in $\Omega_{\boldsymbol{\lambda}, \boldsymbol{z}}$ is no less than $|q(\boldsymbol{\lambda}, c)|$.

Proof. Our proof is parallel to that of [13, Theorem 7.2].

By Proposition 6.1 and Lemma 6.3, the operators $B_{i j}(\boldsymbol{\lambda}, \boldsymbol{z}) \in \operatorname{End}\left(\left(V_{\boldsymbol{\lambda}}\right)^{\mathfrak{g}_{N}}\right)$ are self-adjoint relative to the form $(\cdot, \cdot)_{\boldsymbol{\lambda}, c}$. By Lemma 6.4 ,

$$
\operatorname{dim}\left(\bigcap_{i, j} \bigoplus_{\alpha \in \mathbb{R}}\left(\left(V_{\boldsymbol{\lambda}}\right)^{\mathfrak{g}_{N}}\right)_{B_{i j}(\boldsymbol{\lambda}, \boldsymbol{z})}(\alpha)\right) \geqslant|q(\boldsymbol{\lambda}, c)| .
$$

By Theorem 6.2 , for any $\boldsymbol{\lambda}$ and generic $\boldsymbol{z} \in \stackrel{\circ}{\mathbb{P}}_{n}$ the operators $B_{i j}(\boldsymbol{\lambda}, \boldsymbol{z})$ are diagonalizable and the action of the Bethe algebra $\mathcal{B}$ on $\left(V_{\boldsymbol{\lambda}}\right)^{\mathfrak{g}_{N}}$ has simple spectrum. The same also holds true with $\boldsymbol{\lambda}$ imposed above for any $c$. Thus for generic $\boldsymbol{z}$, the operators $B_{i j}(\boldsymbol{\lambda}, \boldsymbol{z})$ have at least $|q(\boldsymbol{\lambda}, c)|$ common eigenvectors with distinct real eigenvalues, which provides $|q(\boldsymbol{\lambda}, c)|$ distinct real points in $\mathrm{s} \Omega_{\boldsymbol{\lambda}, \boldsymbol{z}}$ by Theorem 5.2. Hence, $d(\boldsymbol{\lambda}, \boldsymbol{z}) \geqslant|q(\boldsymbol{\lambda}, c)|$ for generic $\boldsymbol{z}$, and therefore, for any $\boldsymbol{z}$, due to counting with multiplicities. 
Remark 7.3. If $\operatorname{dim}\left(V_{\boldsymbol{\lambda}}\right)^{\mathfrak{g}_{N}}$ is odd, it follows from Theorem 7.2 by counting parity that

$$
d(\boldsymbol{\lambda}, \boldsymbol{z}) \geqslant|q(\boldsymbol{\lambda}, c)| \geqslant 1 .
$$

Therefore, there exists at least one real point in $\mathrm{s} \Omega_{\boldsymbol{\lambda}, \boldsymbol{z}}$. In particular, if $\operatorname{dim}\left(V_{\boldsymbol{\lambda}}\right)^{\mathfrak{g}_{N}}=1$, then the only point in $s \Omega_{\boldsymbol{\lambda}, \boldsymbol{z}}$ is always real.

The following corollary of Proposition 7.1 and Theorem 7.2 is our main result.

Corollary 7.4. The number $d(\boldsymbol{\lambda}, \boldsymbol{z})$ of real self-dual spaces in $\Omega_{\boldsymbol{\lambda}, \boldsymbol{z}}$ (real points in $\mathrm{s} \Omega_{\boldsymbol{\lambda}, \boldsymbol{z}}$ ) is no less than $|a(\boldsymbol{\lambda}, c)|$, where $a(\boldsymbol{\lambda}, c)$ is the coefficient of the monomial $x_{1}^{N-1} x_{2}^{N-3} \cdots x_{r}^{N+1-2 r}$ in the Laurent polynomial

$$
\Delta_{N} \cdot \prod_{i=1}^{c} \mathcal{S}_{\lambda^{(2 i)}}^{N}\left(x_{1}^{2}, \ldots, x_{r}^{2}\right) \prod_{j=2 c+1}^{n} \mathcal{S}_{\lambda^{(j)}}^{N}\left(x_{1}, \ldots, x_{r}\right) .
$$

Here $\Delta_{N}$ is the Vandermonde determinant of $\mathfrak{g}_{N}$ and $\mathcal{S}_{\lambda^{(s)}}^{N}$ is the Schur function of $\mathfrak{g}_{N}$ associated with $\lambda^{(s)}, s=1, \ldots, n$, see (3.1) and (3.2).

Remark 7.5. Recall that the total number of points (counted with multiplicities) in $\mathrm{s} \Omega_{\boldsymbol{\lambda}, \boldsymbol{z}}$ equals $\operatorname{dim}\left(V_{\boldsymbol{\lambda}}\right)^{\mathfrak{g}_{N}}=q(\boldsymbol{\lambda}, 0)$. Hence if $\boldsymbol{z} \in \mathbb{R}^{\mathrm{P}}{ }_{n}$, Theorem 7.2 claims that all points in $\mathrm{s} \Omega_{\boldsymbol{\lambda}, \boldsymbol{z}}$ are real. It is proved in [15, Corollary 6.3] that for $\boldsymbol{z} \in \mathbb{R P}_{n}$ all points in $\Omega_{\boldsymbol{\lambda}, \boldsymbol{z}}$ are real and multiplicity-free, so are the points in $\mathrm{s} \Omega_{\boldsymbol{\lambda}, \boldsymbol{z}}$.

\subsection{The case $N=2,3$}

Now let us consider the case $N=2,3$. Note that $\operatorname{sGr}(2, d)=\operatorname{Gr}(2, d)$, this case is the usual Grassmannian, which has already been discussed in [13].

Let $N=3$ and $\mathfrak{g}_{3}=\mathfrak{s l}_{2}$. It suffices for us to consider the case that points in $\mathrm{s} \Omega_{\boldsymbol{\lambda}, \boldsymbol{z}}$ have no base points, see the beginning of Section 7.1 for more detail. We shall consider sGr $(3,2 d-1)$ instead of $\operatorname{sGr}(3, d)$, see [10, Section 4.6]. We identify the dominant integral $\mathfrak{s l}_{2}$-weights with nonnegative integers. Let $\boldsymbol{\lambda}=\left(\lambda^{(1)}, \ldots, \lambda^{(n)}\right)$ be a sequence of nonnegative integers and $\boldsymbol{z}=$ $\left(z_{1}, \ldots, z_{n}\right) \in \stackrel{\circ}{\mathbb{P}}_{n}$. Then $\boldsymbol{\lambda}_{A}$ has coordinates

$$
\lambda_{A}^{(s)}=\left(2 \lambda^{(s)}, \lambda^{(s)}, 0\right), \quad s=1, \ldots, n .
$$

We also assume $\left|\boldsymbol{\lambda}_{A}\right|=6(d-2)$.

Recall from [10, Theorem 4.19], if $X \in \mathrm{s} \Omega_{\boldsymbol{\lambda}, \boldsymbol{z}}$, then there exist monic polynomials $\varphi$ and $\psi$ such that $\varphi^{2}, \varphi \psi, \psi^{2}$ form a basis of $X$. Denote by $\sqrt{X}$ the space of polynomials spanned by $\varphi$ and $\psi$. Let $\xi^{(i)}$ be the partitions with at most two parts defined by $\left(\lambda^{(i)}, 0\right), i=1, \ldots, n$. Set $\boldsymbol{\xi}=\left(\xi^{(1)}, \ldots, \xi^{(n)}\right)$, then $|\boldsymbol{\xi}|=2(d-2)$. It follows from the proof of [10, Theorem 4.19] that $\sqrt{X} \in \Omega_{\boldsymbol{\xi}, \boldsymbol{z}} \subset \operatorname{Gr}(2, d)$. The map $\Omega_{\boldsymbol{\xi}, \boldsymbol{z}} \rightarrow \mathrm{s} \Omega_{\boldsymbol{\lambda}, \boldsymbol{z}}$ given by $\sqrt{X} \mapsto X$ is bijective.

Lemma 7.6. The self-dual space $X$ is real if and only if $\sqrt{X}$ is real.

Proof. It is obvious that $X$ is real if $\sqrt{X}$ is real.

Conversely, if $X$ is real, then there exist complex numbers $a_{i}, b_{i}, c_{i}, i=1,2,3$, such that

$$
a_{i} \varphi^{2}+b_{i} \varphi \psi+c_{i} \psi^{2}, \quad i=1,2,3,
$$

are real polynomials and form a basis of $X$. Without loss of generality, we assume $\operatorname{deg} \varphi<\operatorname{deg} \psi$. Since $\operatorname{deg} \varphi<\operatorname{deg} \psi$, we have $c_{i} \in \mathbb{R}, i=1,2,3$. At least one of $c_{i}$ is nonzero. We assume $c_{3} \neq 0$. By subtracting a proper real multiple of $a_{3} \varphi^{2}+b_{3} \varphi \psi+c_{3} \psi^{2}$, we assume further $c_{1}=c_{2}=0$. Continuing with the previous step, we assume that $b_{1}=0, b_{2} \neq 0, a_{1} \neq 0$ and hence obtain that $a_{1}, b_{2}, c_{3} \in \mathbb{R}$. Then $a_{1} \varphi^{2}$ is a real polynomial, so is $\varphi$. Therefore, $a_{2} \varphi+b_{2} \psi$ is also a real polynomial, which implies that the space of polynomials $\sqrt{X}$ is also real. 
Because of Lemma 7.6, the case $N=3$ is reduced to the lower bound for the number of real solutions to osculating Schubert problems of $\operatorname{Gr}(2, d)$, see [13]. Moreover, Corollary 7.4 also applies for this case by putting $N=3, r=1$, and $\mathfrak{g}_{N}=\mathfrak{s l}_{2}$.

\section{Some data for small $N$}

In this section, we give some data obtained from Corollary 7.4 when $N$ is small. Since the cases $N=2,3$ reduce to the cases of [13], we start with $N=4$.

We always assume that $\boldsymbol{\lambda}, \boldsymbol{k}, \boldsymbol{z}$ are invariant under conjugation. By Remark 7.3, we shall only consider the cases that $\operatorname{dim}\left(V_{\boldsymbol{\lambda}}\right)^{\mathfrak{g}_{N}} \geqslant 2$. We also exclude the cases that $\boldsymbol{z} \in \mathbb{R}^{\circ}{ }_{n}$. In particular, the cases that all pairs $\left(\lambda^{(s)}, k_{s}\right), s=1, \ldots, n$, are different.

We write the highest weights in terms of fundamental weights, for example $(1,0,0,1)=$ $\omega_{1}+\omega_{4}$. We also write $\left(\lambda^{(1)}\right)_{k_{1}}, \ldots,\left(\lambda^{(n)}\right)_{k_{n}}$ for $(\boldsymbol{\lambda}, \boldsymbol{k})$ and simply write $\lambda^{(s)}$ for $\left(\lambda^{(s)}\right)_{0}$. We use $\left(\lambda_{1}^{(s)}, \lambda_{2}^{(s)}\right)_{k_{s}}^{\otimes m}$ to indicate that the pair $\left(\left(\lambda_{1}^{(s)}, \lambda_{2}^{(s)}\right), k_{s}\right)$ appears in $(\boldsymbol{\lambda}, \boldsymbol{k})$ exactly $m$ times. For instance, $(0,1)_{1},(0,1)^{\otimes 3}$ represents the pair $(\boldsymbol{\lambda}, \boldsymbol{k})$ where $\boldsymbol{\lambda}=((0,1),(0,1),(0,1),(0,1))$ and $\boldsymbol{k}=(1,0,0,0)$.

\subsection{The case $N=4,5$}

For each $\mathfrak{g}_{4}$-weight $\lambda=\left(\lambda_{1}, \lambda_{2}\right)$, denote by $\lambda_{C}$ the $\mathfrak{g}_{5}$-weight $\left(\lambda_{2}, \lambda_{1}\right)$. Note that $\mathfrak{g}_{4}=\mathfrak{s o}_{5}$ is isomorphic to $\mathfrak{g}_{5}=\mathfrak{s p}_{4}$, the lower bound obtained from the ramification data $\boldsymbol{\lambda}=\left(\lambda^{(1)}, \ldots, \lambda^{(n)}\right)$ and $\boldsymbol{k}=\left(k_{1}, \ldots, k_{n}\right)$ of $\mathfrak{g}_{4}$ is the same as that obtained from the ramification data $\boldsymbol{\lambda}_{\boldsymbol{C}}=$ $\left(\lambda_{C}^{(1)}, \ldots, \lambda_{C}^{(n)}\right)$ and $\boldsymbol{k}=\left(k_{1}, \ldots, k_{n}\right)$ of $\mathfrak{g}_{5}$.

\begin{tabular}{|c|c|c|c|c|}
\hline ramification data & dimension & $c=1$ & $c=2$ & $c=3$ \\
\hline$(0,1)^{\otimes 6}$ & 14 & 2 & 2 & 6 \\
\hline$(1,0)^{\otimes 3},(0,1)^{\otimes 2}$ & 4 & 0,2 & 2 & \\
\hline$(1,0)^{\otimes 3},(1,0)_{1}$ & 3 & 1 & & \\
\hline$(1,0)^{\otimes 4},(0,0)_{1}$ & 3 & 1 & 3 & \\
\hline$(0,2),(0,1)^{\otimes 4}$ & 6 & 0 & 2 & \\
\hline$(0,0)_{1},(0,1)^{\otimes 4}$ & 3 & 1 & 3 & \\
\hline$(1,0),(0,1)^{\otimes 4}$ & 5 & 1 & 1 & \\
\hline$(1,1),(0,1)^{\otimes 3}$ & 2 & 0 & & \\
\hline$(0,1)_{1},(0,1)^{\otimes 3}$ & 3 & 1 & & \\
\hline$(0,2)^{\otimes 2},(0,1)^{\otimes 2}$ & 3 & 1 & 3 & \\
\hline$(1,0)^{\otimes 2},(0,1)^{\otimes 2}$ & 2 & 0 & 2 & \\
\hline$(0,2),(1,0),(0,1)^{\otimes 2}$ & 2 & 0 & & \\
\hline$(1,0)_{1},(1,0),(0,1)^{\otimes 2}$ & 2 & 0 & & \\
\hline$(1,0)^{\otimes 2},(0,1)_{1},(0,1)$ & 2 & 0 & & \\
\hline$(1,1),(1,0)^{\otimes 2},(0,1)$ & 2 & 0 & & \\
\hline
\end{tabular}

Table 1. The case $N=4,5$.

In Table 1, we give lower bounds for the cases from $\operatorname{Gr}(4,7)$ and $\operatorname{Gr}(5,10)$. By the observation above, we transform the case from $\operatorname{Gr}(5,10)$ to its counter part in $\operatorname{Gr}(4, d)$ for some $d$ depending on the ramification data. The number in the column of dimension is equal to $\operatorname{dim}\left(V_{\boldsymbol{\lambda}}\right)^{\mathfrak{g}_{4}}$ for the corresponding ramification data $\boldsymbol{\lambda}$ in each row. The numbers in the column of $c=i$ equal the lower bounds computed from Corollary 7.4 with the corresponding $c$.

For a given $c$, there may exist several choices of complex conjugate pair corresponding to different pairs of $\mathfrak{g}_{N}$-weights. If the corresponding lower bounds are the same, we just write 
one number. For example, in the case of $(0,2)^{\otimes 2},(0,1)^{\otimes 2}$ and $c=1$ of Table 1 , the complex conjugate pair may correspond to the weights $(0,2)^{\otimes 2}$ or $(0,1)^{\otimes 2}$. However, they give the same lower bound 1 . Hence we just write 1 for $c=1$. If the bounds are different, we write the lower bound with the conjugate pairs corresponding to the leftmost $2 c$ weights first while the one with the conjugate pairs corresponding to the rightmost $2 c$ weights last, in terms of the order of the ramification data displayed on each row. Since we have at most 3 cases, the possible remaining case is clear. For instance, in the case $(0,1,0)^{\otimes 4},(0,0,1)^{\otimes 4}$ and $c=2$ of Table 2, the two complex conjugate pairs corresponding to $(0,1,0)^{\otimes 4}$ give the lower bound 12 while the two complex conjugate pairs corresponding to $(0,0,1)^{\otimes 4}$ give the lower bound 24 . The remaining case, where the two conjugate pairs corresponding to $(0,1,0)^{\otimes 2}$ and $(0,0,1)^{\otimes 2}$, gives the lower bound 2 .

\subsection{The case $N=6$}

In what follows, we give lower bounds for ramification data consisting of fundamental weights when $N=6$. We follow the same convention as in Section 8.1.

\begin{tabular}{|c|c|c|c|c|c|}
\hline ramification data & dimension & $c=1$ & $c=2$ & $c=3$ & $c=4$ \\
\hline$(0,0,1)^{\otimes 4}$ & 4 & 0 & 4 & & \\
\hline$(0,1,0)^{\otimes 4}$ & 6 & 2 & 6 & & \\
\hline$(1,0,0)^{\otimes 4}$ & 3 & 1 & 3 & & \\
\hline$(0,0,1)^{\otimes 2},(0,1,0)^{\otimes 2}$ & 3 & 1 & 3 & & \\
\hline$(0,0,1)^{\otimes 2},(1,0,0)^{\otimes 2}$ & 2 & 0 & 2 & & \\
\hline$(0,1,0)^{\otimes 2},(1,0,0)^{\otimes 2}$ & 3 & 1 & 3 & & \\
\hline$(0,0,1)^{\otimes 6}$ & 30 & 2 & 2 & 10 & \\
\hline$(0,1,0)^{\otimes 6}$ & 130 & 8 & 14 & 36 & \\
\hline$(1,0,0)^{\otimes 6}$ & 15 & 3 & 3 & 7 & \\
\hline$(0,1,0)^{\otimes 2},(0,0,1)^{\otimes 4}$ & 34 & 4,2 & 0,6 & 16 & \\
\hline$(0,1,0)^{\otimes 4},(0,0,1)^{\otimes 2}$ & 55 & 3,1 & 3,7 & 19 & \\
\hline$(1,0,0)^{\otimes 2},(0,0,1)^{\otimes 4}$ & 16 & 2 & 0,4 & 10 & \\
\hline$(1,0,0)^{\otimes 4},(0,0,1)^{\otimes 2}$ & 10 & 0,2 & 2,0 & 6 & \\
\hline$(1,0,0)^{\otimes 2},(0,1,0)^{\otimes 4}$ & 46 & 2 & 6 & 18 & \\
\hline$(1,0,0)^{\otimes 4},(0,1,0)^{\otimes 2}$ & 21 & 1,3 & 5,3 & 11 & \\
\hline$(1,0,0)^{\otimes 2},(0,1,0)^{\otimes 2},(0,0,1)^{\otimes 2}$ & 20 & 2 & $0,4,0$ & 10 & \\
\hline$(0,0,1)^{\otimes 8}$ & 330 & 20 & 6 & 0 & 50 \\
\hline$(0,1,0)^{\otimes 8}$ & 6111 & 69 & 59 & 113 & 311 \\
\hline$(1,0,0)^{\otimes 8}$ & 105 & 15 & 9 & 7 & 25 \\
\hline$(0,1,0)^{\otimes 4},(0,0,1)^{\otimes 4}$ & 984 & 22,28 & $12,2,24$ & 0,38 & 108 \\
\hline$(1,0,0)^{\otimes 4},(0,0,1)^{\otimes 4}$ & 116 & 6,12 & $8,2,12$ & 0,10 & 32 \\
\hline$(1,0,0)^{\otimes 4},(0,1,0)^{\otimes 4}$ & 510 & 6,12 & $22,4,18$ & 28,18 & 74 \\
\hline
\end{tabular}

Table 2. The case $N=6$.

\section{Acknowledgements}

The author thanks E. Mukhin and V. Tarasov for useful discussions. The author also thanks the referees for their comments and suggestions that substantially improved the first version of this paper. This work was partially supported by Zhejiang Province Science Foundation, grant No. LY14A010018. 


\section{References}

[1] Borisov L., Mukhin E., Self-self-dual spaces of polynomials, Adv. Math. 190 (2005), 77-118, math.QA/0308128.

[2] Eremenko A., Gabrielov A., Degrees of real Wronski maps, Discrete Comput. Geom. 28 (2002), 331-347, math.AG/0108133.

[3] Feigin B., Frenkel E., Reshetikhin N., Gaudin model, Bethe ansatz and critical level, Comm. Math. Phys. 166 (1994), 27-62, hep-th/9402022.

[4] Frobenius F., Über die Charaktere der symmetrischen Gruppe, König. Preuss. Akad. Wiss. Berlin (1900), 516-534, reprinted in Gessamelte Abhandlungen, Vol. 3, Springer-Verlag, Berlin - New York, 1968, 148-166.

[5] Hein N., Hillar C.J., Sottile F., Lower bounds in real Schubert calculus, São Paulo J. Math. Sci. 7 (2013), 33-58, arXiv:1308.4381.

[6] Hein N., Sottile F., Beyond the Shapiro conjecture and Eremenko-Gabrielov lower bounds, in Lower Bounds Experimental Project, available at http://www.math.tamu.edu/ secant/lowerBounds/lowerBounds.php.

[7] Hein N., Sottile F., Zelenko I., A congruence modulo four in real Schubert calculus, J. Reine Angew. Math. 714 (2016), 151-174, arXiv:1211.7160.

[8] Lu K., Mukhin E., On the Gaudin model of type $\mathrm{G}_{2}$, Commun. Contemp. Math., to appear, arXiv:1711.02511.

[9] Lu K., Mukhin E., Varchenko A., On the Gaudin model associated to Lie algebras of classical types, J. Math. Phys. 57 (2016), 101703, 23 pages, arXiv:1512.08524.

[10] Lu K., Mukhin E., Varchenko A., Self-dual Grassmannian, Wronski map, and representations of $\mathfrak{g l}_{N}, \mathfrak{s p}_{2 r}$, $\mathfrak{s o}_{2 r+1}$, Pure Appl. Math. Q., to appear, arXiv:1705.02048.

[11] Molev A., Feigin-Frenkel center in types B, C and D, Invent. Math. 191 (2013), 1-34, arXiv:1105.2341.

[12] Molev A., Mukhin E., Eigenvalues of Bethe vectors in the Gaudin model, Theoret. and Math. Phys. 192 (2017), 1258-1281, arXiv:1506.01884.

[13] Mukhin E., Tarasov V., Lower bounds for numbers of real solutions in problems of Schubert calculus, Acta Math. 217 (2016), 177-193, arXiv:1404.7194.

[14] Mukhin E., Tarasov V., Varchenko A., The B. and M. Shapiro conjecture in real algebraic geometry and the Bethe ansatz, Ann. of Math. 170 (2009), 863-881, math.AG/0512299.

[15] Mukhin E., Tarasov V., Varchenko A., Schubert calculus and representations of the general linear group, J. Amer. Math. Soc. 22 (2009), 909-940, arXiv:0711.4079.

[16] Mukhin E., Varchenko A., Critical points of master functions and flag varieties, Commun. Contemp. Math. 6 (2004), 111-163, math.QA/0209017.

[17] Pontrjagin L., Hermitian operators in spaces with indefinite metric, Bull. Acad. Sci. URSS. Sér. Math. 8 (1944), 243-280.

[18] Rybnikov L., A proof of the Gaudin Bethe Ansatz conjecture, arXiv:1608.04625.

[19] Soprunova E., Sottile F., Lower bounds for real solutions to sparse polynomial systems, Adv. Math. 204 (2006), 116-151, math.AG/0409504.

[20] Sottile F., Frontiers of reality in Schubert calculus, Bull. Amer. Math. Soc. 47 (2010), 31-71, arXiv:0907.1847. 